\title{
The Effect of Water Absorption on the Dielectric Properties of Polyethylene Hexagonal Boron Nitride Nanocomposites
}

\author{
Raed Ayoob, Fuad Alhabill, Thomas Andritsch, and Alun Vaughan \\ University of Southampton, School of Electronics and Computer Science, Tony Davies High Voltage \\ Laboratory, Southampton, SO17 1 BJ, United Kingdom
}

\begin{abstract}
The effect of water absorption on the dielectric response of polyethylene/hexagonal boron nitride nanocomposites has been studied by dielectric spectroscopy. The nanocomposites have been prepared with hBN concentrations ranging from $2 \mathrm{wt} \%$ to $30 \mathrm{wt} \%$. Fourier Transform infrared (FTIR) spectroscopy and thermogravimetric analysis (TGA) revealed a very small amount of hydroxyl groups on the surface of hBN. Mass loss measurements showed that the nanocomposites did not absorb any water under ambient and dry conditions while there was some water absorption under wet conditions. The dielectric spectroscopy results showed a broad relaxation peak, indicative of different states of water with water shells of different thickness, which moved to higher frequencies with increasing water content. However, the dielectric losses were significantly lower than the losses reported in the literature of nanocomposites under wet conditions. In addition, all the absorbed water was successfully removed under vacuum conditions which demonstrated that the interactions between the water and the nanocomposites were very weak, due to the hydrophobic nature of the hBN surface. This is a highly useful property, when considering these materials for applications in electrical insulation.
\end{abstract}

\section{Introduction}

The use of polymer nanocomposites as materials for electrical insulation has been suggested by Lewis [1], which led to the concept of nanometric dielectrics later popularized under the term nanodielectrics. Lewis proposed that adding nanoparticles into insulating polymers changes the internal charge activity and electrical potential distribution at the nanoparticle/polymer interface, this would results in a nanocomposite with dielectric properties that could not be explained by conventional theories. After Lewis published his paper in 1994, a number of promising experimental results triggered an immense research interest in nanodielectrics within the dielectrics community. Nanodielectrics have since been shown to improve the dielectric breakdown strength [2], reduce space charge accumulation [3], change the nature of the dielectric losses [4], improve partial discharge resistance [5], and reduce the propagation of electrical trees [6]. However, a growing number of publications also pointed out that nanodielectrics can show significant reduction of the breakdown strength, or no change at all [7]-[9]. One culprit for this behavior was soon identified to be adsorbed moisture at the polymer/particle interface.

Dielectric spectroscopy is a useful tool for studying changes in dielectric behavior, which are caused by the effects of nanostructuration. More importantly, dielectric spectroscopy measurements are extremely sensitive to the presence of water. Water has a high real permittivity and can be easily detected, since it readily interacts with a changing electric field. However, published work on the effects of water absorption in nanocomposites is still limited. The available literature has highlighted detrimental effects associated with a range of polymer nanocomposites, which tend to absorb more water in comparison to an unfilled reference polymer. To explain why epoxy/silica nanocomposites absorb up to $60 \%$ more water than the unfilled epoxy, a water shell model has been proposed by Zou et al. [10]. This model suggests, that water molecules surround the surface of the nanoparticles in a few layers when exposed to moisture. Under wet conditions, the dielectric response of a 9 wt $\%$ epoxy/silica nanocomposite indicated quasi-DC conduction, which was attributed to the percolation of water shells around the silica nanoparticles [11]. Similar findings by Hui et al. [12] indicated the presence of a percolating water network in an XLPE/silica nanocomposite containing $12.5 \mathrm{wt} \%$ of silica. In layered nanocomposites, the dependence of water absorption on the aspect ratio of the layered filler has been highlighted by Fabiani et al. [13], where the formation of water shells are more probable in nanocomposites containing high aspect ratio fillers.

This demonstrates one of the biggest challenges faced by nanodielectrics for their widespread adoption in applications: the high tendency of polymer nanocomposites to absorb water due to the hydrophilic nature of the surfaces of nanoparticles. One solution is to modify the surface of nanoparticles, thereby reducing the amount of hydroxyl groups that are available for bonding with water. For example, the work of Lau et al. [14] has shown that different surface treatments of silica significantly reduces the water absorption capabilities in polyethylene/silica nanocomposites, which results in a modified dielectric response with reduced dielectric losses. 
While surface treatment is effective in reducing the amount of available hydroxyl groups, residual hydroxyl groups still remain, which can readily interact with water. Therefore, other methods have to be adopted to remove the surface hydroxyl groups. Calcination is an attractive technique that eliminates the surface hydroxyl groups from hydrophilic nanoparticles and successfully renders the surface of nanoparticles hydrophobic. Indeed, Pallon et al. $[12,13]$ obtained magnesium oxide $(\mathrm{MgO})$ particles by calcination of magnesium hydroxide $\mathrm{Mg}(\mathrm{OH})_{2}$. This resulted, with additional surface treatment, resulted in a hydrophobic nanoparticle with a significantly reduced electrical conductivity, since the hydrophobic nature of $\mathrm{MgO}$ does not increase water absorption. A similar heat treatment approach has been performed by the authors on the surface of zinc oxide $(\mathrm{ZnO})$ particles for use in high voltage insulating applications [17]. Similarly, Praeger et al. [18] showed that calcination of silica resulted in a hydrophobic polyethylene/silica nanocomposite with significantly reduced water absorption and, as a result, reduced dielectric losses. While many efforts have been made to turn the surface of hydrophilic nanoparticles into a hydrophobic nature either by surface treatment or calcination, the use of as-received hydrophobic nanoparticles has been rarely considered. This would provide a faster, cheaper, more straightforward route to material preparation in order to produce hydrophobic, electrically insulating, polymer nanocomposites. In electrically insulating applications, the use of hydrophobic nanoparticles is essential, since the presence of water is highly detrimental to the electrical properties.

Therefore, the work in this study is set out to consider the water absorption behavior of a polymer nanocomposite system, based on a polyethylene matrix incorporating a hydrophobic nanoparticle: hexagonal boron nitride (hBN). Polyethylene was chosen as it is widely used as base material in high voltage power insulation. Hexagonal boron nitride, a layered nanoparticle, is characterized by a highly hydrophobic surface with a basal surface free of hydroxyl groups and edge surfaces composed of a few hydroxyl groups. Its hydrophobic nature, coupled with its highly electrically insulating properties and high thermal conductivity, makes it an attractive choice in electrically insulating composite systems. The objectives of this study were to: characterize the as-received hBN particles by $\mathrm{X}$-Ray diffraction (XRD), investigate the presence of surface hydroxyl groups on the surface of hBN by Fourier Transform Infrared Spectroscopy (FTIR) and thermogravimetric analysis (TGA). The dispersion state within the composites was characterized via scanning electron microscopy (SEM), while the dielectric response and water absorption behavior in polyethylene/hBN nanocomposites were investigated by dielectric spectroscopy (DS).

\section{Experimental}

\subsection{Material}

Low density polyethylene (LDPE LD100BW from ExxonMobil) and high density polyethylene (HD Rigidex HD5813EA from BP Chemicals) were used to prepare the host polymer. The polymer matrix was prepared from a combination of $80 \%$ LDPE and $20 \%$ HDPE blend. The hexagonal boron nitride filler (NX-1 from Momentive) was used in this study. A total of six different materials were prepared by solvent blending, which contained between $2 \mathrm{wt} \%$ and $30 \mathrm{wt} \%$ of hBN, as well as unfilled reference material. A solvent blending procedure was used to prepare all the materials. A total of $10 \mathrm{~g}$ of LDPE and HDPE were initially added to $100 \mathrm{~mL}$ of xylene, followed by simultaneous stirring and heating. After the polymer had dissolved, the mixture was removed from the heat, and the desired amount of hBN, which was dispersed in isopropyl alcohol (IPA) was added. This was followed by continuous, vigorous stirring until the mixture turned into a viscous waxy solid. The resultant mixture was left to dry in a fume cupboard for $7 \mathrm{~d}$, followed by drying in a vacuum oven for $3 \mathrm{~d}$ oven at $60{ }^{\circ} \mathrm{C}$ to remove the residual solvent.

\subsection{Sample Preparation and Conditioning}

Samples for scanning electron microscopy and dielectric spectroscopy were prepared in a melt press at $180{ }^{\circ} \mathrm{C}$ using a load of 5 ton. The samples were immediately placed from the melt in oil bath at $115^{\circ} \mathrm{C}$ for isothermal crystallisation, followed quenching into water. Samples of $\sim 250 \mu \mathrm{m}$ thickness were prepared for use in most characterization methods. Samples for DS were prepared with a $30 \mathrm{~mm}$ diameter gold layer of approximately 20 $\mu \mathrm{m}$ thickness on both sides, to ensure good electrical contact. The samples are abbreviated as PEBN/X, where "X" denotes the hBN content.

Samples for DS were conditioned as follows, for up to 15 days for each corresponding condition:

- "Ambient": Samples were exposed to laboratory ambient conditions $\left(20 \pm 2{ }^{\circ} \mathrm{C}\right.$ and $\left.50 \pm 20 \% \mathrm{RH}\right)$

- "Dry": Samples were in a vacuum oven, which contains dry desiccant pellets, at room temperature

- "Wet": Samples were completely immersed in water at room temperature.

- "Wet to Dry": These are the wet samples which have been placed in a vacuum oven at room temperature 
The mass of all samples was recorded at regular intervals, until no further mass change could be detected, which was interpreted as having reached saturation.

\subsection{Characterization Methods}

XRD was used to determine the crystal structure of the hBN powder. XRD measurements were collected using a Bruker D2 phaser second generation equipment. A copper detector is used with $\mathrm{Cu} \mathrm{K}_{\alpha}=1.5418 \AA$ and the generated X-rays were filtered using a nickel $\mathrm{K}_{\beta}$ filter to obtain monochromatic X-rays with only $\mathrm{K}_{\alpha}$ wavelength. The beam of electrons was accelerated in the vacuum tube at a potential of $30 \mathrm{kV}$ with a $10 \mathrm{~mA}$ value of current. The $2 \theta$ values were scanned from $5^{\circ}$ to $80^{\circ}$ with a $0.02^{\circ}$ increment.

FTIR spectroscopy was used to further characterize the hBN powder. FTIR measurements of the hBN powder were performed using a Perkin Elmer Spectrum GX spectrometer. The spectral data for the powder was collected for wavenumbers in the range of $400 \mathrm{~cm}^{-1}$ to $4000 \mathrm{~cm}^{-1}$ over 32 scans at $4 \mathrm{~cm}^{-1}$ resolution.

TGA was used to study the mass loss behavior of the hBN particles and to confirm the assumed filler contents of the nanocomposites were correct. A Perkin Elmer 1 TGA with a Perkin Elmer Pyris software was used to perform these measurements. The hBN powder was heated from $40{ }^{\circ} \mathrm{C}$ to $900{ }^{\circ} \mathrm{C}$ at a rate of $20{ }^{\circ} \mathrm{C} / \mathrm{min}$ in air.

A JEOL Model JSM-6500F SEM was used with to examine the h-BN dispersion state in each of the samples with an applied voltage of $15 \mathrm{kV}$. Potassium permanganate was gradually added to an etchant mixture composed of 5:2:1 parts sulphuric acid: phosphoric acid: water. The resulting etching reagent was stirred for 15 minutes without heat to give a $1 \%$ solution. The samples were transferred to empty vials in which $\sim 10 \mathrm{ml}$ of the etchant mixture was added and shaken for 4 hours. The samples were then quenched in a solution of $25 \%$ hydrogen peroxide and 75\% quench mixture, composed of 2:7 parts sulphuric acid: water. Finally, the samples were rinsed in distilled water and then acetone, then left to dry before being gold coated to prevent charge accumulation on the surface of the sample during examination in the SEM.

DS was carried out using a Solartron 1296 dielectric interface in combination with a Schlumberger SI 1260 Impedance/phase gain analyser with a dielectric cell. The sample were placed between two $30 \mathrm{~mm}$ diameter parallel electrodes. The sample holder has a guard ring to reduce fringing effects at the edge of the sample. The thickness and diameter of the samples were measured prior to the test. An AC voltage of $1 \mathrm{~V}$ was applied to the sample with a frequency sweep from $10^{-1} \mathrm{~Hz}$ to $10^{6} \mathrm{~Hz}$ at 8 points per decade and 10 cycle integrations per point.

\section{Results and Discussion}

\subsection{Materials Characterization and Composite Structure}

The X-ray diffraction pattern of the $\mathrm{hBN}$ powder is shown in Figure 1. Several peaks are revealed in the XRD pattern of the $\mathrm{hBN}$ powder centred at $2 \theta$ values of $26.2^{\circ}, 40.0^{\circ}, 42.1^{\circ}, 48.2^{\circ}, 53.0^{\circ}$, and $74.6^{\circ}$ corresponding to the (002), (100), (101), (102), (004), and (110) planes respectively. A well-defined, sharp narrow peak relative to all other peaks is observed in the (002) plane in the XRD spectrum which confirms the crystalline structure of the $\mathrm{hBN}$ powder consisting of a periodic structure composed of well-ordered stacked crystal planes, which correspond to the stacked hBN layers as reported elsewhere $[16,17]$.

The peak in (002) plane corresponds to a d-spacing of $3.40 \AA, 0.340 \mathrm{~nm}$, when calculated by rearranging Bragg's Law:

$$
d=\frac{n \lambda}{2 \sin \theta}
$$




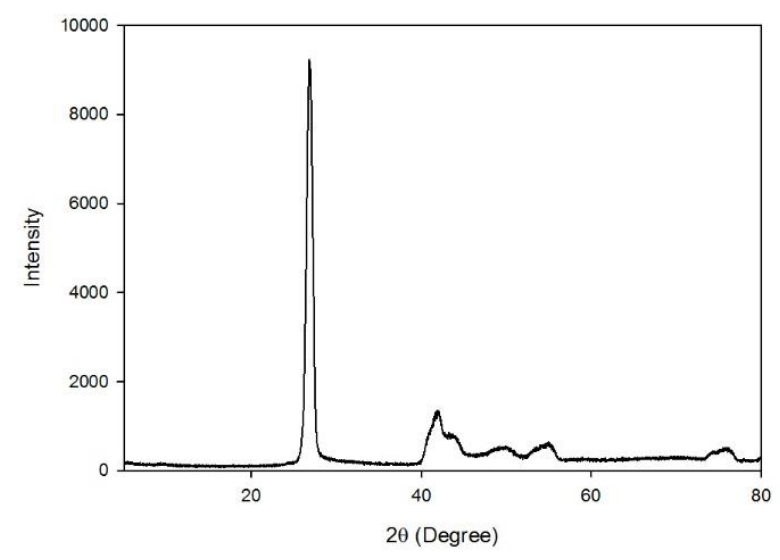

Figure 1: X-Ray diffraction pattern of the hBN powder

Equation (2) is used to measure the lattice constants in a hexagonal structure, where $d_{h k l}$ refers to the " $d$ " spacing in Bragg's Law. Using simple geometry for hexagonal structures, the interlayer spacing $\left(d_{\text {int }}\right)$ and the boronnitrogen bond length $\left(l_{\text {bond }}\right)$ can be determined from the following equation:

$$
\frac{1}{d_{h k l}^{2}}=\frac{4}{3}\left(\frac{h^{2}+h k+k^{2}}{a^{2}}\right)+\frac{l^{2}}{c^{2}}
$$

This yields values of: $\mathrm{a}=0.257 \mathrm{~nm}, \mathrm{c}=0.666 \mathrm{~nm}$ which correspond to $\mathrm{d}_{\mathrm{int}}=0.332 \mathrm{~nm}, \mathrm{l}_{\text {bond }}=0.1484 \mathrm{~nm}$. The vales are consistent with the reported values in the literature $[18,19]$ thereby verifying the hexagonal structure of the boron nitride particles.

The FTIR spectrum of the hBN particles is shown in Figure 2. It shows characteristic peaks of boron nitride in the hexagonal phase, in which the absorption band centered at $1360 \mathrm{~cm}^{-1}$ corresponds to the in-plane stretching of the B-N bonds, while the absorption band centered at $814 \mathrm{~cm}^{-1}$ corresponds to the out-of-plane B-N-B bond bending. These findings are consistent with literature, which indicate these absorption bands are characteristic of the hexagonal phase of boron nitride [20,21]. The in-plane stretching absorption band is centered at a higher wavenumber that the out-of-plane bending due to the stronger covalent bonds between the boron and nitrogen atoms relative to the weaker Van der Waals forces involved in the out-of-plane direction.

Two smaller absorption bands centered at $3131 \mathrm{~cm}^{-1}$ and $3420 \mathrm{~cm}^{-1}$ correspond to the stretching of O-H and N-H bonds, which are found on the edges of the platelets of hBN. However, it is not clear which absorption band corresponds to which type of bond as the absorption bands of hydroxyl and amine groups lie in the same region. Similar characteristic absorption bands of hydroxyl stretching, with a small amount of infrared absorption, in the FTIR spectrum of hBN has been reported elsewhere [20, 21].

Another very sharp absorption band similar, to the $814 \mathrm{~cm}^{-1}$, is centered at $494 \mathrm{~cm}^{-1}$ which has not previously been reported for hBN. Lower absorption bands in the range of $400 \mathrm{~cm}^{-1}$ and $500 \mathrm{~cm}^{-1}$ related to boron based compounds have been reported elsewhere. Medvedev et al. [25] have reported that B-O bonds have absorption bands at $418 \mathrm{~cm}^{-1}$ while Parsons et al. [26] have suggested that absorption bands centered at $\sim 460 \mathrm{~cm}^{-1}$ are characteristic of $\mathrm{B}_{3} \mathrm{O}_{3}(\mathrm{OH})_{3}$ molecules in the structure of orthorhombic metaboric acid. On the other hand, Weltner et al. [27] have reported that absorption bands centered at $\sim 47 \mathrm{~cm}^{-1}$ belong to $\mathrm{B}_{2} \mathrm{O}_{3}$ in boric trioxide As these boron based compounds have absorption bands which are similar to the absorption found in hBN in this study, the boron nitride powder may contain some boron-oxide related impurities in its structure.

Where the FTIR spectrum of hBN is reported in literature, the region below $500 \mathrm{~cm}^{-1}$ is not often included. This region at low wavenumbers is called the "Fingerprint" region which includes very complex molecular bending or deformation vibrations that are often difficult to interpret [28]. 


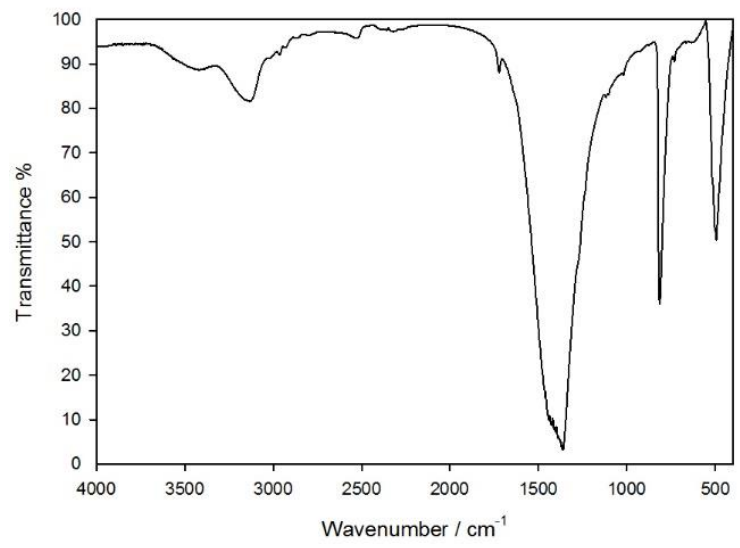

Figure 2: FTIR spectrum of the hBN powder.

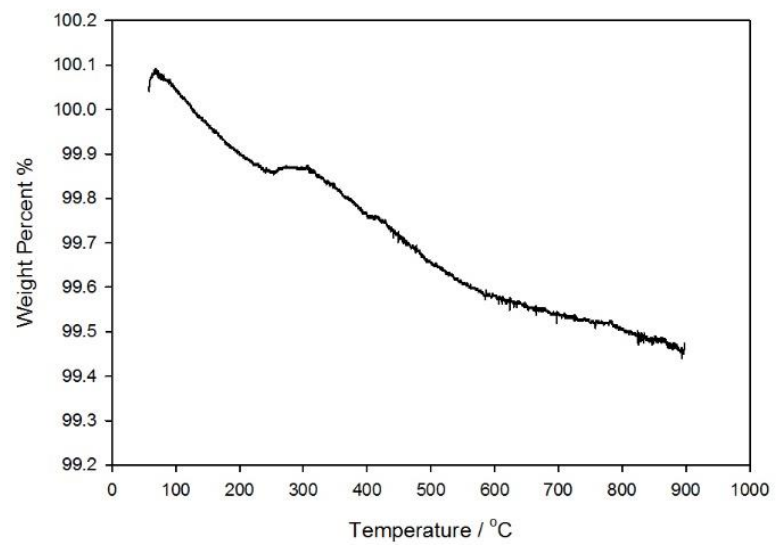

Figure 3: TGA curve for the hBN powder

Figure 3 shows the TGA curve for the hBN powder displaying the weight percent of the powder as a function of temperature. The powder was heated up to $900{ }^{\circ} \mathrm{C}$, where the mass constantly decreased with increasing temperature and experienced a total weight loss of $0.6 \%$ from its initial mass. This mass loss can be attributed to the small amounts of hydroxyl groups which are removed from the surface of the hBN upon heating. The thermal stability of boron nitride has been reported in the past. Huo et al. [29] studied the thermal stability of hBN particles and found that the as-received hBN particles showed no mass change up to $800{ }^{\circ} \mathrm{C}$, while modified hBN particles lost only up to $2 \%$ of mass due to the degradation of silane molecules. Similarly, Yu et al. [20] have reported similar results, where they found that hBN particles remained thermally stable up to $800{ }^{\circ} \mathrm{C}$ with no mass change. In their results, they have plotted TGA curves with a large weight percent scale and so any small mass changes were not observable. Here, a reproducible small mass change was observed which could be considered as insignificant by other researchers when reporting their results.

The thermal stability of boron nitride above $900{ }^{\circ} \mathrm{C}$ has been investigated by a few researchers. Kostoglou et al. [30] reported that hexagonal boron nitride particles remained thermally stable up to $1000{ }^{\circ} \mathrm{C}$. The mass of the hBN particles suddenly increase by $30 \%$ in the temperature range of $1000{ }^{\circ} \mathrm{C}-1200{ }^{\circ} \mathrm{C}$ where it was then stable again. The mass increase is attributed to the oxidation of the boron nitride and the formation of boron trioxide $\left(\mathrm{B}_{2} \mathrm{O}_{3}\right)$ on the surface of $\mathrm{BN}$. The resistance to oxidation, which occurs at very high temperatures, is due to the combination of high crystallinity and small specific surface area which do not provide readily available sites for oxidation. Wang et al. [31] reported similar results where they used hBN particles with different surface areas and found that the onset oxidation temperature decreases and the mass gain percentage increases with increasing surface area of the BN particles. For all particles, the mass of the particles remained constant up to a temperature of $800{ }^{\circ} \mathrm{C}-1000{ }^{\circ} \mathrm{C}$, depending on the surface area of the particles, where a mass gain of up to $35 \%$ was noticed due to the reaction of boron nitride and oxygen to form boron trioxide.

While the boron nitride remained thermally stable up to $900{ }^{\circ} \mathrm{C}$ with an insignificant mass change, other types of particles have been reported to exhibit a completely different behavior. $\mathrm{Li}$ et al. [32] performed TGA measurements on functionalised silica and reported that silica nanoparticles can lose between $13 \%$ and $30 \%$ of weight. Bracho et al. [33] showed that unmodified silica can lose up to $5 \mathrm{wt} \%$ due to the hydroxyl groups on its surface. Jin et al. [34] showed that pure silica can lose up to $18 \%$ of its initial mass due to water content while functionalized silica can lose only up to $8 \%$ of its initial mass. Guo et al. [35] reported that alumina nanoparticles can lose up to $4 \mathrm{wt} \%$ of its initial mass due to the adsorption of moisture and chemical bonding of hydroxyl groups. While oxide based particles show some mass loss upon heating, similar results have been reported for nitride based particles. Tai et al. [36] reported that unmodified silicon nitride nanoparticles lose up to 4 wt $\%$ of its initial mass due to water in the particles while modified silicon nitride particles can lose up to $6 \mathrm{wt} \%$ due to the additional degradation of the coupling agent. Peng et al. [37] showed the unmodified aluminum nitride nanoparticles can lose up to $6 \mathrm{wt} \%$ while modified nanoparticles lose up to $8 \mathrm{wt} \%$ to the decomposition of grafted silane molecules. The mass loss in these type of particles, particularly silica, is much higher than that found in hBN particles, suggesting that boron nitride is highly thermally stable and inherently resistant to moisture absorption.

Figure 4 shows representative SEM micrographs of the $5 \mathrm{wt} \%$ and the $30 \mathrm{wt} \%$ nanocomposites, to illustrate the dispersion state of the $\mathrm{hBN}$, and to link the dispersion state to the level of water absorption. The SEM images reveal a major difference between the two nanocomposites: at low filler contents, the $\mathrm{hBN}$ exists as discrete 
particles in the matrix. In contrast, in nanocomposites with more than $15 \mathrm{wt} \%$, the $\mathrm{hBN}$ exists as a percolating continuous structure throughout the host polymer. In both systems, the hBN particles exist in a range of different sizes and agglomerates. The agglomerate sizes tend to increase with the fillgrade, being the worst in the $30 \mathrm{wt} \%$ nanocomposite. The SEM micrographs will be referred to in terms of percolation effects when the DS results are discussed.
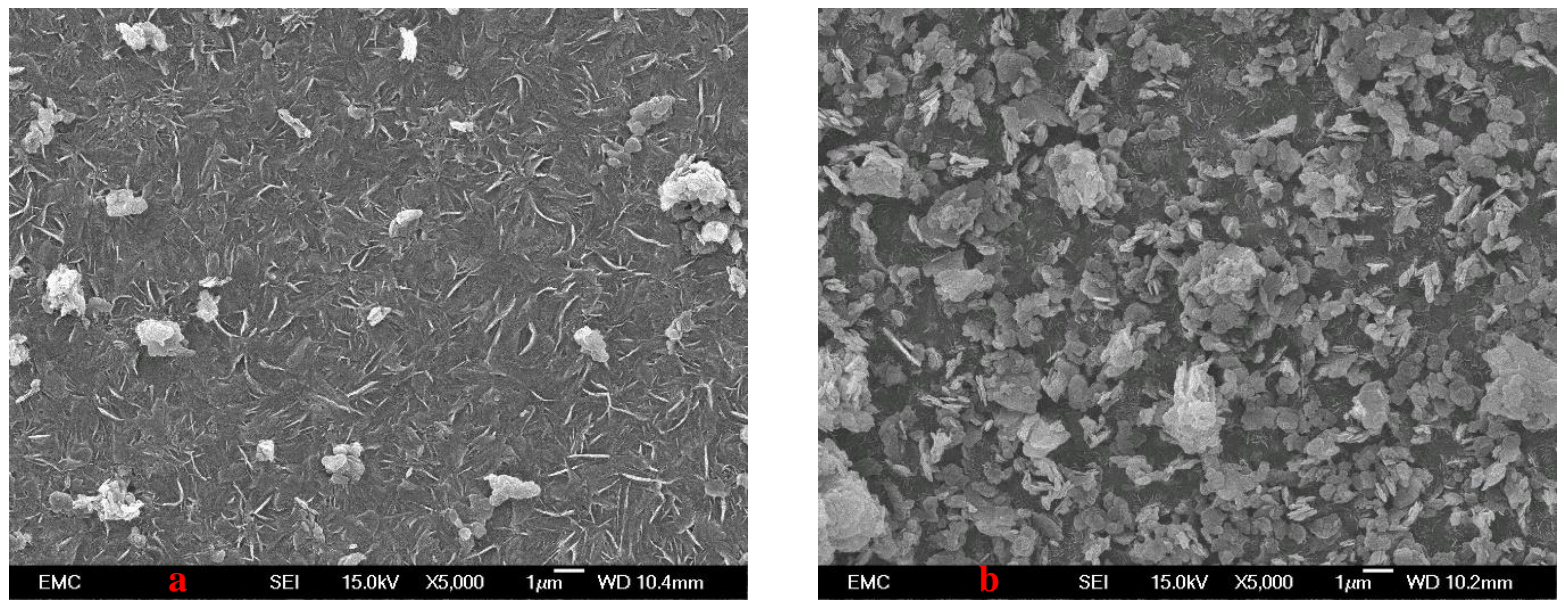

Figure 4: SEM micrographs of (a) $5 \mathrm{wt} \%$ nanocomposite, and (b) $30 \mathrm{wt} \%$ nanocomposite

Table I shows the mass change after saturation in the samples under all conditions. The unfilled PE sample exhibits the same behavior under all conditions, and the lack of significant change in mass suggests that it does not absorb any water. The nanocomposites do not gain or lose significant amounts of mass in "Ambient" and "Dry" conditions respectively, as the mass change in these conditions is less than $0.01 \%$. Since the nanocomposites do not gain any water in "Ambient" conditions and do not have any absorbed water to lose in "Dry" conditions, this suggests that they are completely dry in those two conditions and do not carry significant amounts of water.

Table I: Mass change after conditioning for up to 15 days under respective conditions.

\begin{tabular}{|c|c|c|c|c|}
\hline \multirow{2}{*}{ Material } & \multicolumn{4}{|c|}{ Conditioning Regime } \\
\cline { 2 - 5 } & Ambient & Dry & Wet & Wet to Dry \\
\hline PEBN/0 & $<0.02 \%$ & $<0.02 \%$ & $<0.02 \%$ & $<0.02 \%$ \\
\hline PEBN/2 & $<0.02 \%$ & $<0.02 \%$ & $+0.0239 \%$ & $-0.0221 \%$ \\
\hline PEBN/5 & $<0.02 \%$ & $<0.02 \%$ & $+0.0372 \%$ & $-0.0366 \%$ \\
\hline PEBN/10 & $<0.02 \%$ & $<0.02 \%$ & $+0.0493 \%$ & $-0.0459 \%$ \\
\hline PEBN/20 & $<0.02 \%$ & $<0.02 \%$ & $+0.0667 \%$ & $-0.0684 \%$ \\
\hline PEBN/30 & $<0.02 \%$ & $<0.02 \%$ & $+0.0805 \%$ & $-0.0792 \%$ \\
\hline
\end{tabular}

In "Wet" conditions, the nanocomposites gain some mass due to water and as the hBN content increases, the amount of absorbed water increases accordingly. The mass of the samples immersed in water stops changing after about 2 days. Although the nanocomposites absorb some water, the mass change is insignificant, e.g. the $30 \mathrm{wt} \%$ nanocomposite absorbs only $\sim 0.08 \%$, which is very low compared to e.g. a $10 \mathrm{wt} \% \mathrm{PE}$ silica nanocomposite, which has been shown to absorb more than $1 \%$ of water $[11,36]$. The "Wet to Dry" data clearly indicates that the nanocomposites can lose all the absorbed water, when the "Wet" samples are dried under vacuum conditions, since their masses return to their original values.

\subsection{Dielectric Response}

Figure 5 shows the real and imaginary relative permittivity of all the materials under dry conditions. The figure shows that the real relative permittivity of all materials is frequency independent and increases with increasing hBN content, due to the higher permittivity of the hBN. The imaginary relative permittivity of all materials is characterized by very low losses, and the measurements are within the noise limit of the equipment. 

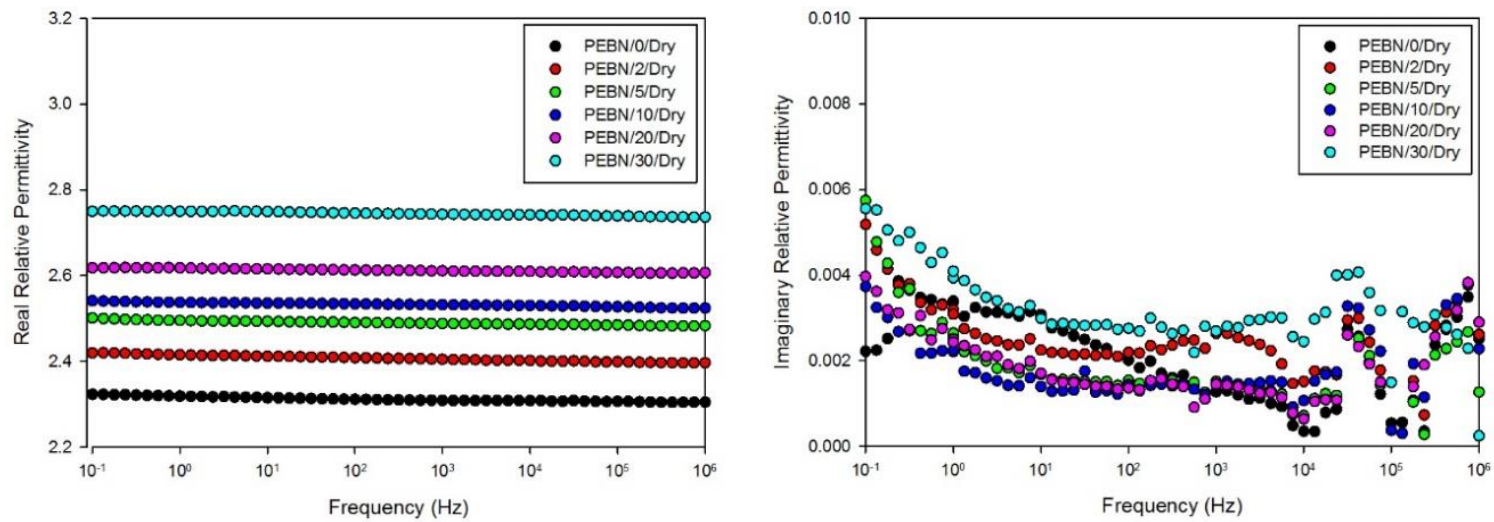

Figure 5: Real relative permittivity and imaginary relative permittivity of the "Dry" samples

The behavior of "Ambient" samples is almost identical to the behavior of the "Dry" sample, no frequency dependent behavior or relaxation peaks could be observed. This suggests that they can both be considered dry for purposes of investigating the dielectric response. Therefore only a representative plot of the "Dry" samples is shown. This result is consistent with lack of the mass change, even after being exposed to conditions with $50 \% \mathrm{RH}$. This is in contrast to $\mathrm{PE}$ based nanocomposites containing silica and silicon nitride particles, which tend to absorb water under ambient conditions [39]. Unlike the hBN nanocomposites in this study, DS of silica and silicon nitride nanocomposites revealed relaxation features, which can be attributed to bound water molecules at the nanoparticle interfaces.

Figure 6 shows the time-dependent real relative permittivity behavior of all the samples under different water immersion times, to investigate the origin of the relaxation peaks. It can be observed that the real relative permittivity behavior of all nanocomposites is similar and the value of the real relative permittivity gradually increases with the immersion time. As more water is absorbed, the quantity of water molecules increases. This increases the amount of dipoles that can readily respond to the applied electric field, which then manifests itself as higher measurable permittivity. This is analogous with published work on the dielectric response of PE nanocomposites with silica and silicon nitride particles under wet conditions $[11,36]$. However, the measured changes in permittivity due to water absorption is much lower in data presented here. This is attributed to the different surface states of the fillers: the hydrophilic surface of silica readily absorbs water, whereas the hydrophobic surface of hBN does not favor water absorption.

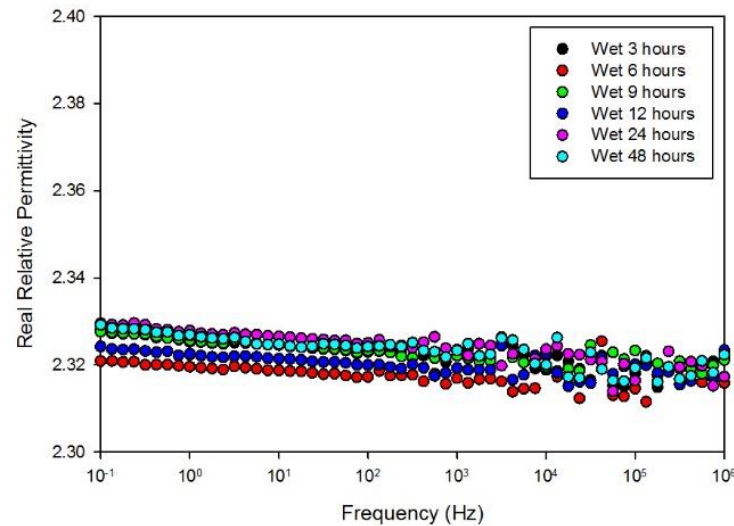

(a)

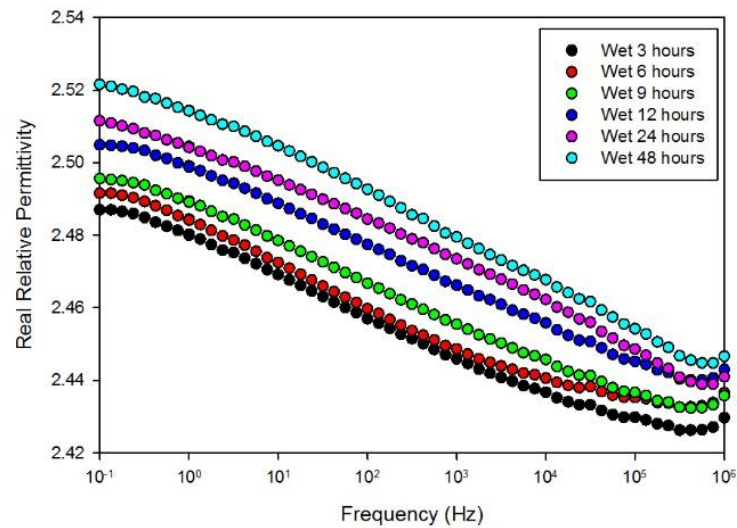

(b) 


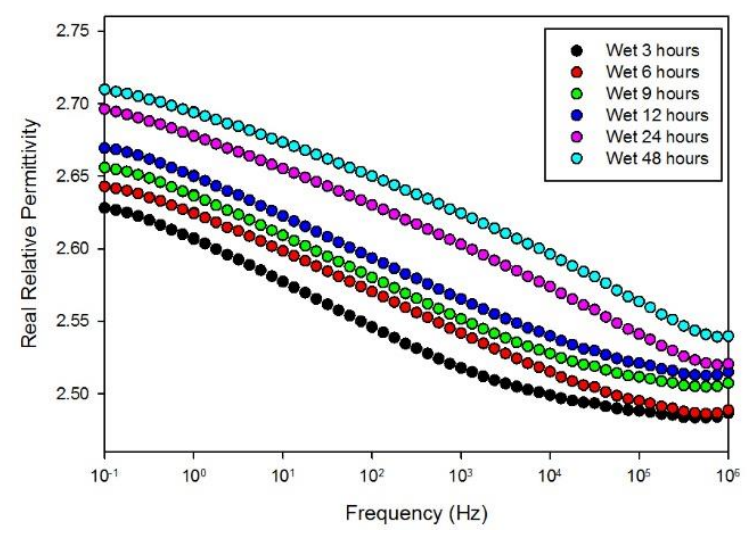

(c)

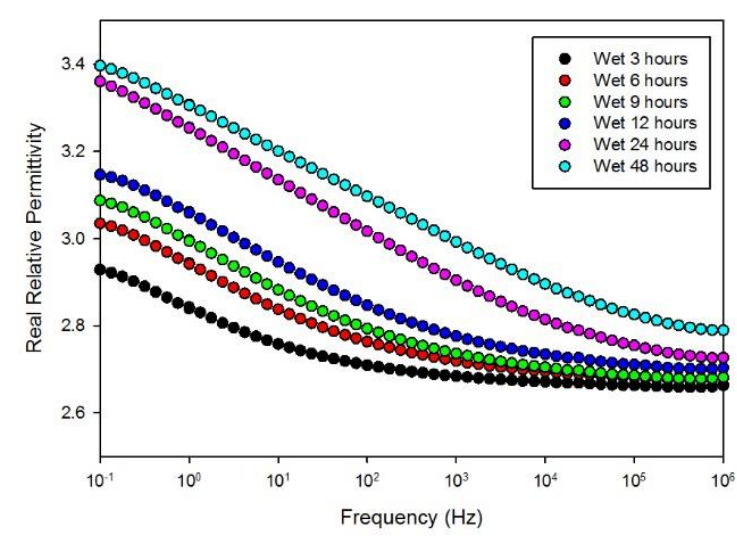

(e)

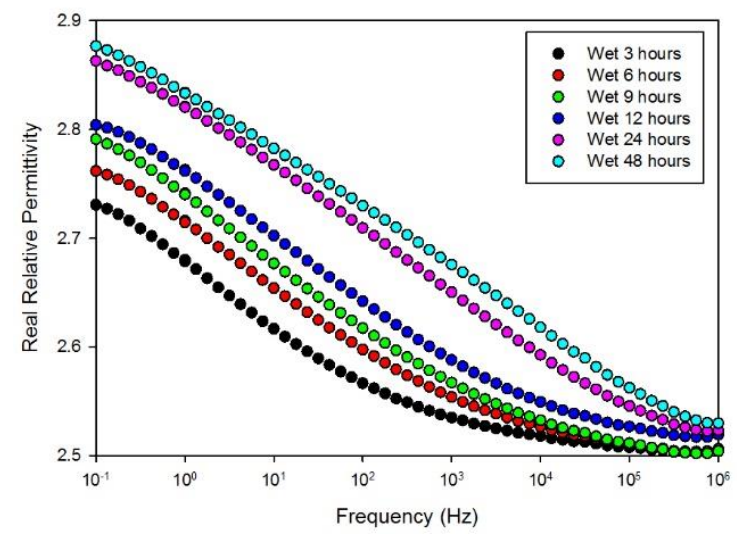

(d)

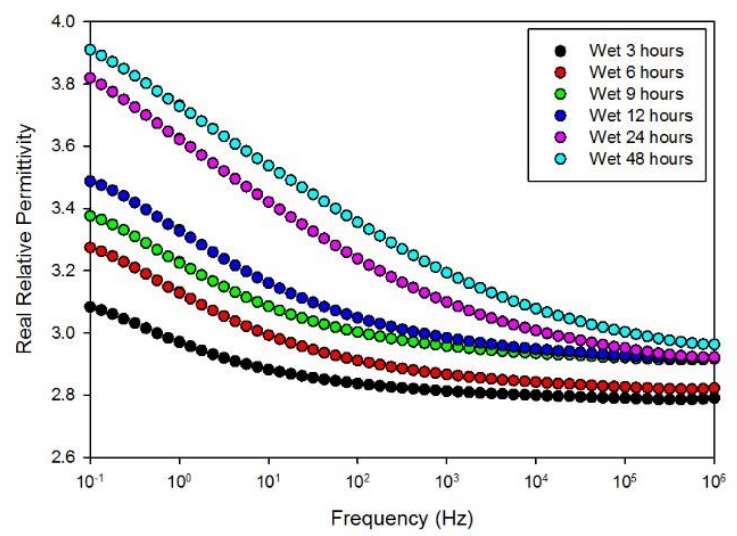

(f)

Figure 6: Real relative permittivity of the "Wet" polyethylene nanocomposites containing (a) 0 wt \%, (b) 2 wt \%, (c) $5 \mathrm{wt} \%$, (d) $10 \mathrm{wt} \%$, (e) $20 \mathrm{wt} \%$, (f) $30 \mathrm{wt} \%$ of hBN.

Figure 7 shows the time-dependent imaginary relative permittivity behavior of all the samples under different water immersion times. Considering the nanocomposites containing $10 \mathrm{wt} \% \mathrm{of} \mathrm{hBN}$ and less, a broad loss peak can be observed, which shifts to higher frequencies as the immersion time increases, and the intensity of this loss peak increases with increasing hBN content. This is clearer in the $5 \mathrm{wt} \%$ and $10 \mathrm{wt} \%$ nanocomposite than in the $2 \mathrm{wt}$ $\%$ due to larger quantity of absorbed water. The effect of water absorption on the dielectric response of poly(ethylene-co-butyl acrylate)/alumina nanocomposites [40], polyethylene/silica nanocomposites [14], and polyethylene/silicon nitride nanocomposites [39] have shown that the dielectric losses increase with increasing filler content, with loss peaks shifting to higher frequencies with increasing water content. The broad loss peaks in the imaginary relative permittivity are in line with the increase in real relative permittivity across the entire measured frequency range which, confirms the existence of many different states of water in the system. This has been highlighted in the work of Zou et al. [11] who developed a water shell model to describe the dielectric properties of epoxy/silica nanocomposites under wet conditions. The existence of different states of water suggests that water layers with different thicknesses exist around the hBN particles, which correspond to relaxations at different frequencies due to the different mobility of these layers. This is consistent with the SEM images, which show uniform yet agglomerated structures in the nanocomposites. Here hBN particles exist in different sizes in the PE with different interfacial regions, which in turn will develop different states of water.

The imaginary relative permittivity behavior of the $20 \mathrm{wt} \%$ and $30 \mathrm{wt} \%$ nanocomposites appears to be significantly different from the nanocomposites containing $10 \mathrm{wt} \% \mathrm{of} \mathrm{hBN}$ content and less, which suggests different mechanisms are in effect in the nanocomposites containing high loading levels of hBN particles. The imaginary relative permittivity of the highly loaded nanocomposites increases at low frequencies, and its magnitude increases with increasing water absorption, whereas the imaginary relative permittivity of the nanocomposites with low loading levels decreases at low frequencies to exhibit the broad relaxation peaks. 


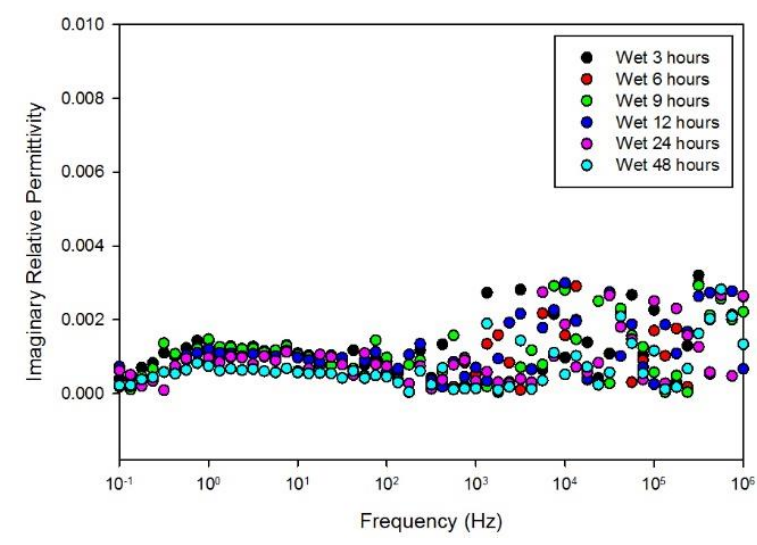

(a)

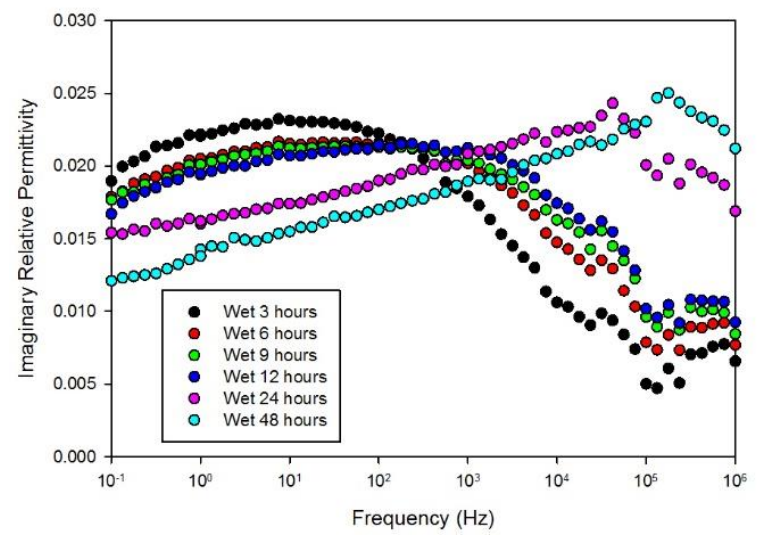

(c)

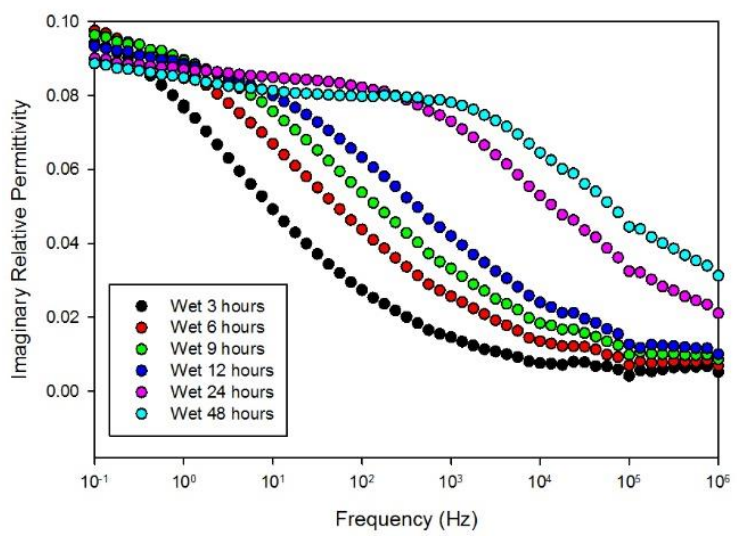

(e)

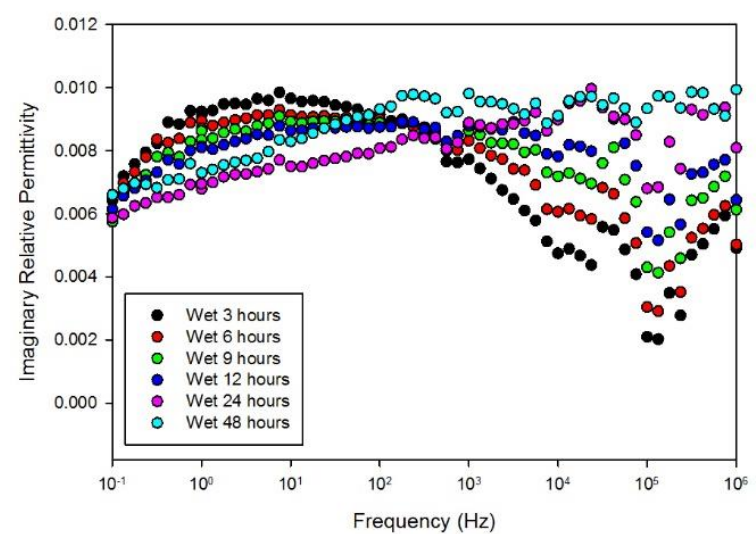

(b)

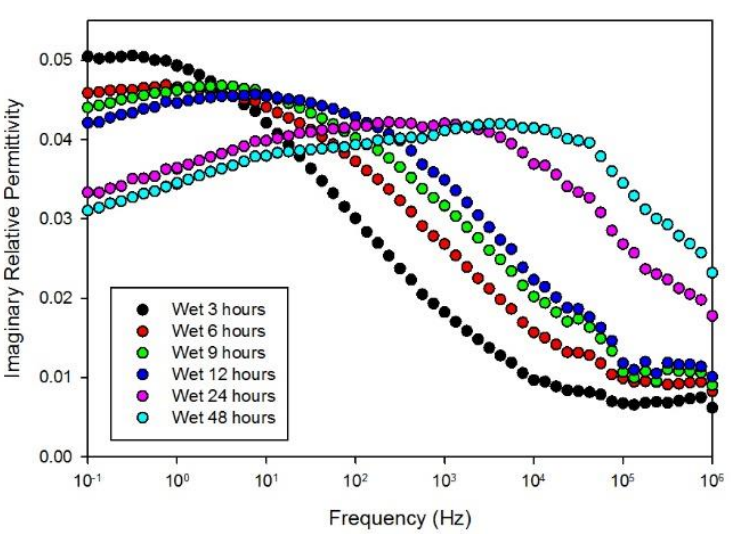

(d)

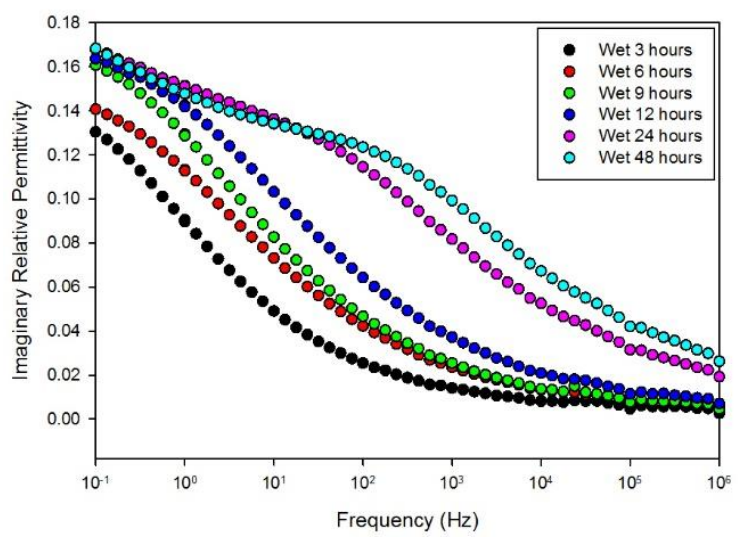

(f)

Figure 7: Imaginary relative permittivity of the "Wet" polyethylene nanocomposites containing (a) 0 wt \%, (b) 2 wt $\%$, (c) $5 \mathrm{wt} \%$, (d) $10 \mathrm{wt} \%$, (e) $20 \mathrm{wt} \%$, (f) $30 \mathrm{wt} \%$ of hBN.

The increase in real relative permittivity and the upturn in the imaginary relative permittivity at low frequencies, which is more evident in the $30 \mathrm{wt} \%$ nanocomposite, suggests there is a percolating water network which facilitates electrical conduction. This behavior has been reported by Hui et al. [12], who observed a different dielectric response in silica XLPE nanocomposites containing low silica content, in comparison to the nanocomposites containing a high silica content. Their dielectric spectroscopy results also show mid-frequency loss peaks in the 5 wt $\%$ nanocomposites due to the bound water. This is different to the behavior of the $12.5 \mathrm{wt} \%$ nanocomposite in which the imaginary permittivity increases with decreasing frequency with a low frequency upturn due to water percolation at high filler content. Similarly, these features have been reported in $9 \mathrm{w} \%$ epoxy/silica nanocomposites 
containing a percolating network of water at high relative humidity levels [11], which absorb a significantly larger amount of water than the unfilled epoxy.

The increase in the imaginary permittivity with decreasing frequency has been previously described as a consequence of quasi-DC conduction due to the formation of conductive paths. This is usually observed when the slopes of the real and imaginary permittivity are parallel with a slope of -1 in the log-log plot of the imaginary permittivity with frequency which has been observed in nanocomposites containing a percolating water network $[8,9,37]$. A log-log plot of the real and imaginary permittivity of the $20 \mathrm{wt} \%$ and the $30 \mathrm{wt} \%$ is shown in Figure 8 which shows a flat behavior, with slopes of -0.0253 and -0.0531 respectively, which is clearly different from -1 . This suggest that, contrary to expectations, no percolating water network exists in these systems. This is all the more surprising, since a percolating structure of $\mathrm{hBN}$ particles can be observed in the high magnification SEM micrographs (Figure 4).

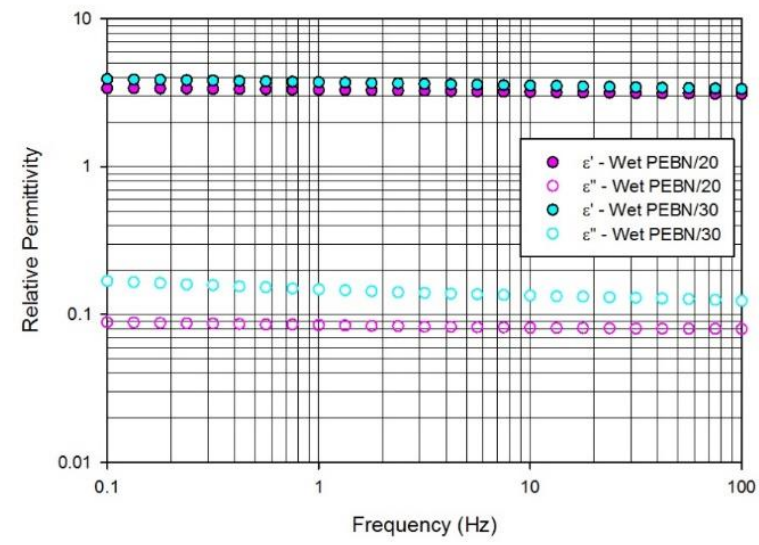

Figure 8: Log-log plot of the relative permittivity of the "Wet" $20 \mathrm{wt} \%$ and $30 \mathrm{wt} \%$ polyethylene nanocomposites

Percolating water networks at much lower filler loading levels have been reported in [11], [12], [39]. These systems have incorporated spherical particles, rather than layered platelets. This suggests that the nature of water shells in layered and spherical particles are significantly different. Fabiani et al studied the effect of water absorption on nanocomposites containing layered fillers with different aspect ratios [13]. They used a polyethylene-covinylacetate polymer with a high aspect ratio fluorohectorite filler (aspect ratio > 100) and a low aspect ratio boehmite filler (aspect ratio 1). The real permittivity behavior of the wet fluorohectorite sample is similar to the wet hBN samples in our case. However, the permittivity for a wet $5 \mathrm{wt} \%$ fluorohectorite sample increased by $87 \%$, whereas the permittivity for the wet $5 \mathrm{wt} \% \mathrm{hBN}$ sample in our study increased by only $7 \%$. The dielectric loss spectrum in the wet fluorohectorite sample show a broad peak in the low frequency region, corresponding to tightly bound water at the polymer/filler interface. However, the behavior of the boehmite wet sample is not very different from the dry sample. They noticed that the electrical properties (including space charge accumulation, breakdown strength, and conductivity) were much worse in the fluorohectorite sample than in the boehmite sample. They explained this by the presence of water shells that can interconnect easier in particles with a higher aspect ratio which can create a percolation path between water shells, depending on the filler and water content. This contradicts the findings in this study which revealed that a percolating water network is not readily formed in hBN layered nanocomposites.

While percolation is achieved at much filler loading levels in nanocomposites containing layered fillers rather than spherical fillers, the formation of a percolating water network could be determined by a combination of both the aspect ratio and the surface chemistry of the filler rather just one factor. Indeed, the study of Fabiani et al. [13] has considered the presence of hydroxyl groups around all the surfaces of the fluorohectorite filler which easily resulted in a percolating water network. In contrast, the hBN particles are characterized by hydrophobic basal surfaces, free of hydroxyl groups, with slightly hydrophilic edge surfaces as a result of a small amount of hydroxyl groups on the edges due to manufacturing defects, which has been confirmed by the TGA results of the hBN particles. In fact, many attempts have been made to create a stable hydrophilic hBN surface by grafting hydroxyl groups on the edges of the hBN platelets [41]. This just highlights the inherent hydrophobic nature of the hBN particles, as they are used in this work. This suggests that water clusters form around the edges of the hBN platelets, rather than completely surrounding the hBN particles. It would serve to limit the formation of a percolating water network. This is also validated by the mass change measurements, which confirm that only low amounts of water has been absorbed, in comparison to systems shown in literature that use other particle types. 
The slight upturn at lower frequencies, which has been discussed earlier as not a cause of water percolation, could be leading to a much broader peak at much lower frequencies. Nanocomposites that had been immersed in water were found to easily lose water to the surrounding air under ambient conditions. Therefore DS measurements at lower frequencies than $0.01 \mathrm{~Hz}$ could provide misleading data, as the measurements would require in excess of three hours. A "wet" $30 \mathrm{wt} \%$ nanocomposite can lose up to $10 \%$ of the absorbed water when exposed to ambient conditions for this duration. Therefore, measurements at lower frequencies would not accurately represent the dielectric behavior of the material.

The same upturn at lower frequencies was shown in the $10 \mathrm{wt} \%$ nanocomposite that was immersed in water for 3 hours, which later appeared as a broad peak at higher frequencies after further immersion in water. The same mechanism could be in effect in the highly loaded nanocomposites, where this peak could be located at much lower frequencies. A peak at lower frequencies could be due to a number of reasons. For example, slow relaxations due to the stronger bonding of water to the hydroxyl groups, since larger amounts of such groups are available for bonding with increasing hBN content. Alternatively, the water molecules could be physically restrained within the large hBN aggregates, which consequently hinders the motion of these water molecules. The physical confinement of water molecules results in a slower reorientation, which is manifested as a very low frequency relaxation peak. While the overlapping of water clusters between some neighboring hBN particles or hBN aggregates should not be excluded, the existence of a percolating water network forming complete conducting paths between the two electrodes is, however, unlikely.

Figure 7 (e) and Figure 7 (f) also show the existence of distinct mid-frequency relaxation features after 24 hours of water immersion, which appear as a result of interfacial polarization of bound water molecules at the polyethylene/hBN interface, and move to higher frequencies with increasing water content. These distinct peaks only appear after 24 hours of water immersion, as these peaks could have been at frequencies lower than the measured frequency range when the samples were immersed in water for less than 24 hours. It is interesting to note that the broad loss peak of the $30 \mathrm{wt} \%$ nanocomposite, which contains more water, is at lower frequency than the broad loss peak of the $20 \mathrm{wt} \%$ nanocomposite which seems to be contradictory as more water generally implies a larger amount of mobile species which in turn leads to higher frequency relaxations. However, this is due to the different nature of the bound water molecules and their interaction with the different amounts of available hBN particles implying the water molecules are more tightly bound in the $30 \mathrm{wt} \%$ nanocomposite thus restricting the mobility of the water. Nevertheless, the dielectric losses are larger in the $30 \mathrm{wt} \%$ sample especially at lower frequencies, although much smaller than the losses observed in polyethylene nanocomposites containing silica or silicon nitride particles even at $10 \mathrm{wt} \%$ or less [39], therefore confirming the hydrophobic nature of boron nitride which less prone to absorbing water.

Praeger et al. [18] reported a very similar behavior in wet polyethylene nanocomposites containing $10 \mathrm{wt} \%$ calcined silica where a small relaxation peak appears at $\sim 10^{4} \mathrm{~Hz}$ in the imaginary permittivity which increases with decreasing frequency leading to a broader low frequency relaxation peak centered around $\sim 1 \mathrm{~Hz}$. The wet hBN nanocomposites in this study could have a similar behavior, with the mechanisms discussed previously, where the low frequency peak at $1 \mathrm{~Hz}$ in the wet $10 \mathrm{wt} \%$ polyethylene/calcined silica nanocomposite is located at much lower frequencies in the $20 \mathrm{wt} \%$ and $30 \mathrm{wt} \%$ nanocomposites below $0.1 \mathrm{~Hz}$.

The effects of water on the real and imaginary relative permittivity, which have been previously observed, are completely eliminated when the "Wet" samples have been completely dried is identical to the "Dry" behavior is therefore not shown. After drying, the behavior of all nanocomposites becomes similar to the "Dry" and "Ambient" samples once again. The values and behavior of the real and imaginary relative permittivity have been reduced to the same values as in the "Dry" and "Ambient" where they have become constant and frequency independent once again. When the "Wet" nanocomposite samples have been dried, their real and imaginary relative permittivity values and behavior have once again become identical to the "Dry" and "Ambient" samples, and have lost all the mass they previously gained due to the amount of water they absorbed. The results from mass measurements and complex permittivity suggest that the water absorbed in the nanocomposites is free water that is not tightly bound at the nanoparticle/polymer interface thus it was easily removed by drying. Since boron nitride absorbs a small amount of water, any amount of absorbed water will be loosely bound. When water is tightly bound to the surface of the hBN particles, its effects will appear in the dielectric loss measurements at lower frequencies whereas the effects of loosely bound water are apparent at higher frequencies due to the higher mobility of the loosely bound water molecules. It has been shown that polyethylene nanocomposites contain both tightly and loosely bound water which are manifested in the imaginary relative permittivity as two distinct relaxation peaks at low and high frequencies respectively $[11,37]$. If any tightly bound water molecules do exists in the system investigated in this study, their relaxation peak would be at a very low frequency lower than the minimum measured frequency of 0.1 $\mathrm{Hz}$. It can then be concluded that any water related relaxation features observed in the dielectric response is attributed to loosely bound water molecules held together with the hydroxyl groups by weak Van der Waals forces. 
Not many studies have been done on the effect of water absorption in boron nitride based nanocomposites due to their hydrophobic nature. Unlike the systems shown in this study which are only based on boron nitride, most studies in literature on the effects of water absorption have been done on nanocomposites with silica or other oxide based fillers. Zhang et al. [42] showed that the inclusion of nanoalumina in both epoxy and polyethylene produced a low frequency loss peak attributed to the tightly bound water. They suggested that the absorbed water accumulates at different sites in the nanocomposites than the pure epoxy due to the large interfacial surfaces when the nano particles are introduced. Some researchers have attempted to turn hydrophilic particles into a hydrophobic state by either changing the surface chemistry of the nano particles through surface functionalization or through calcination, where the hydroxyl groups are completely eliminated which fundamentally changes the water absorption kinetics and the dielectric response due to the changed interfacial region. For example, the work of Lau et al. [14] has shown that the permittivity and dielectric losses in polyethylene silica nanocomposites were reduced by changing the interface when the hydroxyl groups on the surface of silica were substituted with the non-polar propyl $\left(\mathrm{C}_{3} \mathrm{H}_{7}\right)$ groups that do not respond to the electric field. Furthermore, their work has shown that the nanocomposites containing propyl surface groups absorb a much smaller amount of water than the untreated nanocomposites containing mostly surface hydroxyl groups which in turn alters the dielectric response. This highlights the importance of the surface chemistry of the nanoparticles which changes the interfacial region around them and thus affecting the amount of water that can be absorbed by the material.

\section{Conclusion}

The dielectric response of the unfilled polyethylene is unaffected by any type of conditioning due to the hydrophobic nature of the polyethylene polymer. The dielectric response of the "Ambient" and "Dry" nanocomposites is very similar as the nanocomposites do not absorb any water under ambient conditions. Under ambient and dry conditions, the real relative permittivity of the nanocomposites is frequency independent and increases with increasing $\mathrm{hBN}$ content. The imaginary relative permittivity is frequency independent and within the noise limit of the equipment, which suggested that the nanocomposites exhibit very low losses under these conditions. The dielectric response of the "Wet" nanocomposites is significantly different than the "Ambient" and "Dry" nanocomposites despite absorbing a small amount of water. The measurements showed that the nanocomposites exhibit a broad relaxation peak due to the presence of water, which shifts to higher frequencies with increasing water content. While the nanocomposites absorbed a very small amount of water even at high hBN content, this behavior was nevertheless associated with dielectric losses at most of the studied frequency spectrum. However, these losses, even in the $30 \mathrm{wt} \%$ nanocomposites, are by far negligible when compared to the studies in literature consisting of nanoparticles with a much less filler content. This study has also shown that the nanocomposites were able to lose all the amount of absorbed water after drying which revealed that the interactions between the $\mathrm{hBN}$ and water are very weak, due to the very small amount of available hydroxyl groups. It is important to understand the nature of the interaction between the materials and water before designing any material for use in high voltage applications. The work in this study has clearly revealed the weak nature of the interactions between water and polyethylene/hBN nanocomposites, which is a desired property for electrical insulation applications as strong interactions could highly lead to undesirable electrical properties. The materials produced in this study have proven to be very promising electrically insulating materials due to their extreme hydrophobicity. Therefore, when considering polymer nanocomposites for electrical insulation applications, it is necessary to choose an extremely as-received hydrophobic particle instead of going through the particle processing, i.e. surface treatment or calcination, route. As the dielectric spectroscopy measurements suggested promising results, the dependence of electrical properties such as dielectric breakdown strength and electrical conductivity on water would be the next step to explore the full potential of these materials.

\section{References}

[1] T. J. Lewis, "Nanometric dielectrics," IEEE Transactions on Dielectrics and Electrical Insulation, vol. 1, no. 5, pp. 812-825, 1994.

[2] M. Roy, J. K. Nelson, R. K. MacCrone, and L. S. Schadler, "Candidate mechanisms controlling the electrical characteristics of silica/XLPE nanodielectrics,” Journal of Materials Science, vol. 42, no. 11, pp. 3789-3799, 2007.

[3] J. K. Nelson and J. C. Fothergill, "Internal Charge Behaviour of Nanocomposites.," Nanotechnology, vol. 15, no. 5, pp. 1-28, 2004. 

vol. 12 , no. 5, pp. 914-928, Oct. 2005.

[6] M. G. Danikas and T. Tanaka, "Nanocomposites - A review of electrical treeing and breakdown," IEEE Electrical Insulation Magazine, vol. 25, no. 4, pp. 19-25, 2009.

[7] T. Andritsch, R. Kochetov, Y. T. Gebrekiros, P. H. F. Morshuis, and J. J. Smit, "Short term DC breakdown strength in epoxy based BN nano- and microcomposites," IEEE International Conference on Solid Dielectrics, pp. 1-4, 2010.

[8] C. D. Green, A. S. Vaughan, G. R. Mitchell, and T. Liu, "Structure Property Relationships in Polyethylene / Montmorillonite Nanodielectrics," IEEE Transactions on Dielectrics and Electrical Insulation, vol. 15, no. 1, pp. 134-143, 2008.

[9] K. Y. Lau, A. S. Vaughan, G. Chen, and I. L. Hosier, "Polyethylene nanodielectrics: The effect of nanosilica and its surface treatment on electrical breakdown strength," Conference on Electrical Insulation and Dielectric Phenomena, pp. 21-24, Oct. 2012.

[10] C. Zou, J. C. Fothergill, and S. W. Rowe, “A 'Water Shell’ Model for the Dielectric Properties of Hydrated Silica-filled Epoxy Nano-composites," International Conference on Solid Dielectrics, pp. 389-392, 2007.

[11] C. Zou, J. C. Fothergill, and S. W. Rowe, "The effect of water absorption on the dielectric properties of epoxy nanocomposites," IEEE Transactions on Dielectrics and Electrical Insulation, vol. 15, no. 1, pp. 106-117, 2008.

[12] L. Hui, L. S. Schadler, and J. K. Nelson, "The Influence of Moisture on the Electrical Properties of Crosslinked Polyethylene Silica Nanocomposites," IEEE Transactions on Dielectrics and Electrical Insulation, vol. 20, no. 2, pp. 641-653, 2013.

[13] D. Fabiani, G. Montanari, and L. Testa, "Effect of aspect ratio and water contamination on the electric properties of nanostructured insulating materials," IEEE Transactions on Dielectrics and Electrical Insulation, vol. 17, no. 1, pp. 221-230, 2010.

[14] K. Y. Lau, a S. Vaughan, G. Chen, I. L. Hosier, and a F. Holt, "On the dielectric response of silica-based polyethylene nanocomposites,” Journal of Physics D: Applied Physics, vol. 46, no. 9, p. 095303, 2013.

[15] L. K. H. Pallon, a T. Hoang, a M. Pourrahimi, M. S. Hedenqvist, F. Nilsson, S. Gubanski, U. W. Gedde, and R. T. Olsson, "The impact of $\mathrm{MgO}$ nanoparticle interface in ultra-insulating polyethylene nanocomposites for high voltage DC cables," Journal of Materials Chemistry A, vol. 4, no. 22, pp. 8590-8601, 2016.

[16] L. K. H. Pallon, R. T. Olsson, D. Liu, a M. Pourrahimi, M. S. Hedenqvist, a T. Hoang, S. Gubanski, and U. W. Gedde, "Formation and the structure of freeze-dried $\mathrm{MgO}$ nanoparticle foams and their electrical behaviour in polyethylene," Journal of Materials Chemistry A, vol. 3, no. 14, pp. 7523-7534, 2015.

[17] a. M. Pourrahimi, D. Liu, V. Ström, M. S. Hedenqvist, R. T. Olsson, and U. W. Gedde, "Heat treatment of ZnO nanoparticles: new methods to achieve high-purity nanoparticles for high-voltage applications," J. Mater. Chem. A, vol. 3, no. 33, pp. 17190$17200,2015$.

[18] M. Praeger, I. L. Hosier, a S. Vaughan, and S. G. Swingler, "The effects of surface hydroxyl groups in polyethylene-silica nanocomposites," in IEEE Electrical Insulation Conference, 2015, pp. 201-204.

[19] Y. Lin, T. Williams, T. Xu, W. Cao, H. Elsayed-Ali, and J. Connell, "Aqueous dispersions of few-layered and monolayered hexagonal boron nitride nanosheets from sonication-assisted hydrolysis: critical role of water," The Journal of Physical Chemistry $C$, vol. 115 , pp. 2679-2685, 2011.

[20] B. Yu, W. Xing, W. Guo, S. Qiu, W. Xin, S. Lo, and Y. Hu, "Thermal exfoliation of hexagonal boron nitride for effective enhancements on thermal stability, flame retardancy and smoke suppression of epoxy resin nanocomposites via sol-gel process," Journal of Materials Chemistry A, vol. 4, pp. 7330-7340, 2016.

[21] M. Xu, T. Liang, M. Shi, and H. Chen, "Graphene-like two-dimensional materials.," Chemical reviews, vol. 113, no. 5, pp. 376698, May 2013

[22] D. Golberg, Y. Bando, Y. Huang, T. Terao, M. Mitome, and C. Tang, "Boron nitride nanotubes and nanosheets," ACS Nano, vol. 4, no. 6, pp. 2979-2993, 2010.

[23] M. T. Huang and H. Ishida, "Surface study of hexagonal boron nitride powder by diffuse reflectance Fourier transform infrared spectroscopy," Surface and Interface Analysis, vol. 37, no. 7, pp. 621-627, 2005. 
[24] C. Gautam, C. S. Tiwary, S. Jose, G. Brunetto, S. Ozden, S. Vinod, P. Raghavan, S. Biradar, D. S. Galvao, and P. M. Ajayan, "Synthesis of Low-Density, Carbon-Doped, Porous Hexagonal Boron Nitride Solids," ACS Nano, vol. 9, no. 12, pp. 12088$12095,2015$.

[25] E. F. Medvedev and a. S. Komarevskaya, "IR spectroscopic study of the phase composition of boric acid as a component of glass batch," Glass and Ceramics, vol. 64, pp. 42-46, 2007.

[26] J. L. Parsons, "Vibrational Spectra of Orthorhombic Metaboric Acid,” The Journal of Chemical Physics, vol. 33, no. 6, pp. 1860$1866,1960$.

[27] W. Weltner and J. Warn, "Matrix Isolation of High-Temperature Vapors: Boric Oxide," The Journal of Chemical Physics, vol. 37, no. 2, pp. 292-303, 1962.

B. Stuart, Infrared Spectroscopy: Fundamentals and Applications. John Wiley \& Sons Inc., 2004.

[29] J. Hou, G. Li, N. Yang, L. Qin, M. E. Grami, Q. Zhang, N. Wang, and X. Qu, "Preparation and Characterization of Surface Modified Boron Nitride Epoxy Composites with Enhanced Thermal Conductivity," RSC Advances, vol. 4, pp. 44282-44290, 2014.

[30] N. Kostoglou, K. Polychronopoulou, and C. Rebholz, "Thermal and chemical stability of hexagonal boron nitride (h-BN) nanoplatelets," Vacuum, vol. 112, pp. 42-45, 2015.

[31] L. Wang, R. Hang, Y. Xu, C. Guo, and Y. Qian, "From ultrathin nanosheets, triangular plates to nanocrystals with exposed (102) facets, a morphology and phase transformation of sp2 hybrid BN nanomaterials," RCS Advances, vol. 4, no. 27, pp. 14233-14240, 2014.

[32] W. Li, Y. Xu, Y. Zhou, W. Ma, S. Wang, and Y. Dai, "Silica nanoparticles functionalized via click chemistry and ATRP for enrichment of $\mathrm{Pb}$ (II) ion," Nanoscale Research Letters, vol. 7, no. 1, p. 1, 2012.

[33] D. Bracho, V. N. Dougnac, H. Palza, and R. Quijada, "Functionalization of silica nanoparticles for polypropylene nanocomposite applications," Journal of Nanomaterials, vol. 2012, 2012.

[34] H. Jin, P. H. F. Morshuis, J. J. Smit, and T. Andritsch, "The effect of surface treatment of silica nanoparticles on the breakdown strength of mineral oil," IEEE International Conference on Dielectric Liquids, pp. 1-4, 2014

[35] Z. Guo, T. Pereira, O. Choi, Y. Wang, and H. T. Hahn, "Surface functionalized alumina nanoparticle filled polymeric nanocomposites with enhanced mechanical properties," Journal of Materials Chemistry, vol. 16, no. 27, p. 2800, 2006.

[36] Y. Tai, J. Miao, J. Qian, R. Xia, and Y. Zhang, "An effective way to stabilize silicon nitride nanoparticles dispersed in rubber matrix by a one-step process," Materials Chemistry and Physics, vol. 112, no. 2, pp. 659-667, 2008.

[37] W. Peng, X. Huang, J. Yu, P. Jiang, and W. Liu, "Electrical and thermophysical properties of epoxy/aluminum nitride nanocomposites: Effects of nanoparticle surface modification," Composites Part A: Applied Science and Manufacturing, vol. 41, no. 9, pp. 1201-1209, 2010.

[38] I. L. Hosier, M. Praeger, a S. Vaughan, and S. G. Swingler, "Electrical properties of polymer nano-composites based on oxide and nitride fillers," Electrical Insulation Conference, no. June, pp. 438-441, 2015.

[39] I. L. Hosier, M. Praeger, A. S. Vaughan, and S. G. Swingler, "The effects of water on the dielectric properties of silicon based nanocomposites," IEEE Transactions on Nanotechnology, vol. PP, no. 99, pp. 1-10, 2016.

[40] N. Jäverberg, H. Edin, P. Nordell, S. Nawaz, H. Hillborg, B. Azhdar, and U. W. Gedde, "Dielectric properties of alumina-filled poly (ethylene-co-butyl acrylate) nanocomposites Part II- wet studies," IEEE Transactions on Dielectrics and Electrical Insulation, vol. 19, no. 2, pp. 391-399, 2012.

[41] Q. Weng, X. Wang, X. Wang, Y. Bando, and D. Golberg, "Functionalized hexagonal boron nitride nanomaterials: emerging properties and applications," Chemical Society Reviews, vol. 45, pp. 3989-4012, 2016.

[42] C. Zhang and G. C. Stevens, "The dielectric response of polar and non-polar nanodielectrics," IEEE Transactions on Dielectrics and Electrical Insulation, vol. 15, no. 2, pp. 606-617, 2008. 